\title{
RECTILINEAR OSCILLATIONS OF A RIGID SPHEROID IN AN ELASTIC MEDIUM*
}

BY S. DATTA (Lucknow University) AND R. P. KANWAL (Pennsylvania State University)

1. Introduction. A method of singularities has recently been developed to solvevarious displacement-type boundary value problems in elastostatics [1]. Our aim is toextend this method to elastodynamics. Specifically, we study the dynamical displacements set up in an infinite elastic space when a rigid spheroid, embedded in this space, is subjected to a transverse periodic displacement. Since there are two lengths in this problem, namely, the major axis $a$ and the minor axis $b$, there arise two parameters; one is $m_{a}=\left(\rho \omega^{2} a^{2} / \mu\right)^{1 / 2}$, and the other is $m_{b}=\left(\rho \omega^{2} b^{2} / \mu\right)^{1 / 2}$, where $\rho$ is the density of the medium, $\omega$ is the circular frequency and $\mu$ is the shear modulus. Previously, one of the authors has studied various boundary value problems of this nature $[2,3]$. However, it was assumed that these parameters are of the same order of magnitude. In the present study, when we take both parameters into account we not only recover the known result as a limiting case but get various other interesting limits as well.

The analysis is based on the suitable distributions of the fundamental solutions of the partial differential equations involved and the matched asymptotic expansions [1, 2, 4].

2. Mathematical formulation. Let a rigid prolate spheroid $S$,

$$
\left(x^{2} / a^{2}\right)+\left(r^{2} / b^{2}\right)=1 ; \quad r^{2}=y^{2}+z^{2}, \quad c^{2}=\left(a^{2}-b^{2}\right)=e^{2} a^{2},
$$

where $e(0 \leq e<1)$ is the eccentricity and $2 c$ is the focal length, embedded in an isotropic and homogeneous elastic space, oscillate along the $y$-axis so that the displacement is $d_{0} \exp$ $(i \omega t)$. The Navier equations of elastodynamics are

$$
(\lambda+\mu) \nabla \nabla \cdot \mathrm{U}+\mu \nabla^{2} \mathrm{U}-\rho \frac{\partial^{2} \mathrm{U}}{\partial t^{2}}=0
$$

where $\mathrm{U}$ is the displacement vector, $\lambda, \mu$ are Lamés constants and $\rho$ is the density of the medium. This equation is to be solved subject to the boundary conditions

$$
\mathbf{U}=d_{0} \mathbf{e}_{y} \exp (i \omega t) \text { on } S, \quad \mathrm{U} \rightarrow 0, \text { at } \infty,
$$

where $\mathbf{e}_{y}$ is the unit vector along $y$-axis. It is convenient to introduce a new vector $\mathbf{u}$ such that

$$
\mathbf{u}=d_{0} \mathbf{e}_{y} \exp (i \omega t)-\mathbf{U} .
$$

Then the system of equations (2) are modified as follows:

$$
\begin{gathered}
(\lambda+\mu) \nabla \cdot \nabla \mathbf{u}+\mu \nabla^{2} \mathbf{u}-\rho \frac{\partial^{2} \mathbf{u}}{\partial t^{2}}-\rho \omega^{2} d_{0} \mathbf{e}_{y} \exp (i \omega t)=0, \\
\mathbf{u}=0 \text { on } S, \quad \mathbf{u} \rightarrow d_{0} \mathbf{e}_{y} \exp (i \omega t) \text { at } \infty .
\end{gathered}
$$

To obtain the inner expansion we introduce the inner variables

* Received June 30, 1977; revised version received December 24, 1977. 


$$
x^{\prime}=x / a, \quad y^{\prime}=y / b, \quad z^{\prime}=z / b, \quad \mathbf{u}^{\prime}=\left(\mathbf{u} / d_{0}\right) \exp (-i \omega t),
$$

so that Eq. $(3 a)$ becomes

$$
\begin{gathered}
\frac{1}{1-2 \nu}\left[\frac{b}{a} \mathbf{e}_{x} \frac{\partial}{\partial x^{\prime}}+\mathbf{e}_{y} \frac{\partial}{\partial y^{\prime}}+\mathbf{e}_{z} \frac{\partial}{\partial z^{\prime}}\right]\left[\frac{b}{a} \mathbf{e}_{x} \frac{\partial}{\partial x^{\prime}}+\mathbf{e}_{y} \frac{\partial}{\partial y^{\prime}}+\mathbf{e}_{z} \frac{\partial}{\partial z^{\prime}}\right] \cdot \mathbf{u}^{\prime} \\
+\left[\frac{b^{2}}{a^{2}} \frac{\partial^{2}}{\partial x^{\prime 2}}+\frac{\partial^{2}}{\partial y^{\prime 2}}+\frac{\partial^{2}}{\partial z^{\prime 2}}\right] \mathbf{u}^{\prime}+m_{b}{ }^{2}\left(\mathbf{u}^{\prime}-\mathbf{e}_{y}\right)=0
\end{gathered}
$$

where $\mathbf{e}_{x}$ and $\mathbf{e}_{z}$ are unit vectors in the $x$ and $z$ directions respectively, $\nu=\lambda / 2(\lambda+\mu)$ is Poisson's ratio and $m_{b}^{2}=\rho \omega^{2} b^{2} / \mu$. This analysis is based on the assumption that $b$ is small and $m_{b}<<1$.

In order to obtain the solution for small $m_{b}$ we take the inner expansion as

$$
\mathbf{u}^{\prime}=\mathrm{u}_{0}^{\prime}+\mathrm{u}_{1}^{\prime}+\mathrm{u}_{2}^{\prime}+\cdots,
$$

such that $\mathbf{u}_{n+1}^{\prime} / \mathbf{u}_{n}^{\prime} \rightarrow 0$, as $m_{b} \rightarrow 0$. The equations for $\mathbf{u}_{0}^{\prime}$ and $\mathbf{u}_{1}^{\prime}$, in dimensional space variables, are

$$
\frac{1}{1-2 \nu} \nabla \nabla \cdot \mathbf{u}_{0}^{\prime}+\nabla^{2} \mathbf{u}_{0}=0, \quad \frac{1}{1-2 \nu} \nabla \nabla \cdot \mathbf{u}_{1}+\nabla^{2} \mathbf{u}_{1}^{\prime}=0 .
$$

These equations are to be solved subject to the inner conditions

$$
\mathrm{u}_{0}^{\prime}=\mathrm{u}_{1}^{\prime}=0 \text { on } S \text {, }
$$

along with the requirement that the inner expansion must match the outer expansion.

To obtain the solutions in the farfield it is convenient to introduce the following two sets of outer variables:

$$
\begin{aligned}
\tilde{x}=M_{b} x / b, & \tilde{y}=M_{b} y / b, & \tilde{z}=M_{b} z / b, \\
x^{*}=m_{b} x / b, & y^{*}=m_{b} y / b, & z^{*}=m_{b} z / b,
\end{aligned}
$$

where $M_{b}^{2}=((1-2 \nu) /(2-2 \nu)) m_{b}^{2}$ and $M_{b}$ and $m_{b}$ are of the same order of magnitude and no distinction will be made in writing $M_{b} \rightarrow 0$ or $m_{b} \rightarrow 0$. Next, we introduce the outer expansion

$$
\mathbf{u}^{\prime}=\mathbf{e}_{y}+\mathbf{q}_{1}+\mathbf{q}_{2}+\cdots,
$$

where $\mathbf{q}_{n+1} / \mathbf{q}_{n} \rightarrow 0$, as $m_{b} \rightarrow 0$, and set

$$
\mathrm{q}_{1}=M_{b} \tilde{\nabla} h_{1}+m_{b} \nabla^{*} \times \mathbf{g}_{1},
$$

where $\tilde{\nabla}$ and $\nabla^{*}$ are the gradient operators in the coordinates $(9 a)$ and $(9 b)$ respectively. When we express Eq. (5) in terms of outer variables (9) and substitute the expansion (10) in them we obtain

$$
\tilde{\nabla}^{2} h_{1}+h_{1}=0, \quad \nabla^{* 2} \mathbf{g}_{1}+\mathbf{g}_{1}=0 .
$$

Since $\mathbf{u}^{\prime} \rightarrow \mathbf{e}_{y}$ at infinity, it follows that $\mathbf{q}_{1} \rightarrow 0$ at infinity. Furthermore, this solution should match the inner solution. Finally, let us introduce two new dimensionless parameters $M_{a}$ $=(a / b) M_{b}$ and $m_{a}=(a / b) m_{b}$, so that $M_{a}$ and $m_{a}$ are small and are of the same order of magnitude:

$$
M_{a}^{2}=\frac{1-2 \nu}{2-2 \nu} m_{a}^{2} ; \quad m_{a}^{2}=\frac{\rho \omega^{2} a^{2}}{\mu} ; \quad 0<m_{a} \ll<1, \quad M_{a}=0\left(m_{a}\right) .
$$


3. The solution. We use the singularity method in [1] and solve Eqs. (7) subject to a zero displacement condition. Indeed, it follows from the analysis in [1] that

$$
\begin{aligned}
\mathbf{u}_{0}^{\prime} & =\frac{\alpha}{\alpha_{0}}\left[\mathbf{e}_{y}-\alpha_{0} \int_{-a e}^{a e}\left\{\frac{3-4 \nu}{R_{\xi}} \mathbf{e}_{y}+\frac{y \mathbf{R}_{\xi}}{R_{\xi}^{3}}\right\} d \xi\right. \\
& \left.-\beta_{0} \int_{-a e}^{a e}\left(a^{2} e^{2}-\xi^{2}\right) \nabla\left\{\frac{\partial}{\partial y}\left(\frac{1}{R_{\xi}}\right)\right\} d \xi\right],
\end{aligned}
$$

where

$$
\begin{aligned}
& R_{\xi}=(x-\xi) \mathbf{e}_{x}+y \mathbf{e}_{y}+z \mathbf{e}_{z}, \quad R_{\xi}=\left|\mathbf{R}_{\xi}\right|, \\
& \alpha_{0}=-\frac{2 e^{2} \beta_{0}}{1-e^{2}}=2 e^{2}\left[2 e-\left(1-7 e^{2}+8 \nu e^{2}\right) L\right]^{-1}, \quad L=\log \frac{1+e}{1-e},
\end{aligned}
$$

and $\alpha$ is, as yet, an unknown constant. From the above relation it follows that $\beta_{0}=0\left(b^{2}\right)$. Accordingly, the outer limit of $\mathbf{u}_{0}^{\prime}$ is given as

$$
\lim _{R^{\prime} \rightarrow \infty} \mathbf{u}_{0}^{\prime}=\frac{\alpha}{\alpha_{0}}\left[\mathbf{e}_{y}-\alpha_{0} \int_{-e M_{a}}^{e M_{a}}\left\{\frac{3-4 \nu}{\tilde{R}_{\xi}} \mathbf{e}_{y}+\frac{\tilde{y} \mathbf{R}_{\xi}}{\tilde{R}_{\xi}^{3}}\right\} d \tilde{\xi}+O\left(\frac{1}{R^{\prime 3}}\right)\right],
$$

where $R^{\prime 2}=\left(x^{\prime 2}+y^{\prime 2}+z^{\prime 2}\right), \tilde{\xi}=\left(M_{b} / b\right) \xi, \tilde{R_{\xi}}=\left|\mathbf{R}_{\xi}\right|, \mathbf{R}_{\xi}=\left(M_{b} / b\right) \mathbf{R}_{\xi}$.

Case 1. $M_{a}$ small. In our entire discussion, $M_{b}$ is assumed to be always small. Now we take $M_{a}$ also to be small so that

$$
0<M_{b} \leq M_{a} \ll 1, \quad M_{b}=O\left(M_{a}\right) .
$$

Then we can reduce relation (16) still further. Indeed,

$$
\lim _{R^{\prime} \rightarrow \infty, M_{a} \rightarrow 0} \mathbf{u}_{0}^{\prime}=\frac{\alpha}{\alpha_{0}}\left[\mathbf{e}_{y}-2 e M_{a} \alpha_{0}\left\{\frac{3-4 \nu}{\mathbf{R}} \mathbf{e}_{y}+\frac{\tilde{y} \hat{\mathbf{R}}}{\tilde{R}^{3}}\right\}+O\left(M_{a}^{2}, \frac{M_{a}}{R^{2}}\right)\right],
$$

where $\mathbb{R}=\tilde{x} \mathbf{e}_{x}+\tilde{y} \mathbf{e}_{y}+\tilde{z} \mathbf{e}_{z}, \tilde{R}=|\mathbb{R}|$. Since the limit of $\mathbf{u}_{0}^{\prime}$ as given by (17) must match the outer solution (10) we have in the first approximation

$$
\alpha=\alpha_{0} \text {. }
$$

Next, we express $q_{1}$ in terms of a line distribution (of constant density) of fundamental solutions of the partial differential equations (12) so that

$$
\begin{aligned}
\tilde{\mathbf{q}}_{1}= & B^{*} \int_{-e m_{a}}^{e m_{a}}\left[-\frac{\exp \left(-i R_{\xi}^{*}\right)}{R_{\xi}^{*}} \mathbf{e}_{y}+\nabla^{*} \frac{\partial}{\partial{ }^{*}}\left\{\frac{1}{R_{\xi}^{*}}-\frac{\exp \left(-i R_{\xi}^{*}\right)}{R_{\xi}^{*}}\right] d \xi^{*}\right. \\
& -\tilde{B} \int_{-e M_{a}}^{e M_{a}} \tilde{\nabla} \frac{\partial}{\partial \tilde{y}}\left\{\frac{1}{\tilde{R}_{\xi}}-\frac{\exp \left(-i \tilde{R_{\xi}}\right)}{\tilde{R_{\xi}}}\right\} d_{\tilde{\xi}},
\end{aligned}
$$

where $\xi^{*}=\left(M_{b} / b\right) \xi, \mathbf{R}_{\xi}^{*}=\left(M_{b} / b\right) \mathbf{R}_{\xi}, R_{\xi}^{*}=\left(\mathbf{R}_{\xi}^{*}\right)$, and the constants $B^{*}$ and $\tilde{B}$ are to be determined from matching conditions. Indeed, the inner limit of $\mathbf{q}_{1}$ is

$$
\begin{aligned}
\lim _{\tilde{R} \rightarrow 0 M_{a} \rightarrow 0} \mathrm{q}_{1} & =2 e M_{a}\left[-\frac{B^{*}+\tilde{B}}{2 \tilde{R}} \mathbf{e}_{y}-\frac{\left(B^{*}-\tilde{B}\right)}{2} \frac{\tilde{y} \tilde{R}}{\tilde{R}^{3}}\right. \\
& \left.+\frac{i}{3}\left\{2\left(\frac{2-2 \nu}{1-2 \nu}\right)^{1 / 2} B^{*}+\tilde{B}\right\} \mathbf{e}_{y}\right]+O\left(M_{a}^{2}, M_{a} \tilde{R}\right) .
\end{aligned}
$$

Matching the inner limit of the quantity $\mathbf{e}_{y}+\mathrm{q}_{1}$ from the above relation with the outer 
limit (17), we have

$$
B^{*}=4 \alpha_{0}(1-\nu), \quad \tilde{B}=2 \alpha_{0}(1-2 \nu) .
$$

The third term on the right side of $(20)$ is to be matched with the outer limit of $\mathbf{u}_{1}^{\prime}$. Since Eq. ( $7 a$ ) and the boundary condition (8) satisfied by $\mathbf{u}_{1}^{\prime}$ are the same as those satisfied by $u_{0}^{\prime}$, we take

$$
\mathrm{u}_{1}^{\prime}=A \mathrm{u}_{0}^{\prime}, \quad A=\text { constant. }
$$

Matching the third term in (20) with the outer solution of $\mathbf{u}_{1}^{\prime}$, which can be easily written down by the help of (17), we get

$A=\frac{2 e M_{a} i}{3}\left\{2\left(\frac{2-2 \nu}{1-2 \nu}\right)^{1 / 2} B^{*}+\tilde{B}\right\}=\frac{8}{3} e(1-\nu) \alpha_{0} m_{a}\left\{2+\left(\frac{1-2 \nu}{2-2 \nu}\right)^{3 / 2}\right\} i$.

Next, we add the contributions of the Kelvin solutions distributed between the foci [1] and easily find the dynamic force as

$$
\begin{aligned}
P & =16 \pi \mu d_{0}(1-\nu) \exp (i \omega t) \int_{-a e}^{a e} \alpha_{0}\left[1+\frac{8 i}{3} e(1-\nu) \alpha_{0}\left\{2+\left(\frac{1-2 \nu}{2-2 \nu}\right)^{3 / 2}\right\} m_{a}+O\left(m_{a}^{2}\right)\right] d \xi \\
& =32 \pi \mu d_{0} a e(1-\nu) \alpha_{0}\left[1+\frac{8 i}{3} e(1-\nu) \alpha_{0}\left\{2+\left(\frac{1-2 \nu}{2-2 \nu}\right)^{3 / 2}\right\} m_{a}\right] \exp (i \omega t)+O\left(m_{a}^{2}\right) \\
& =P_{0}\left[1+\frac{P_{0} i}{12 \pi \mu d_{0} a}\left\{2+\left(\frac{1-2 \nu}{2-2 \nu}\right)^{3 / 2}\right\} m_{a}\right] \exp (i \omega t)+O\left(m_{a}^{2}\right)
\end{aligned}
$$

where

$$
P_{0}=64 \pi \mu(1-\nu) d_{0} a e^{3}\left[2 e-\left(1-7 e^{2}+8 \nu e^{2}\right) L\right]^{-1}
$$

is the static force evaluated in [1]. Relation (24) agrees with the value obtained earlier [1, 2] except that now the value of $P_{0}$ is given explicitly.

Case 2. $M_{a}$ arbitrary, $M_{b} \ll 1$. In this case the outer limit of $\mathbf{u}_{0}^{\prime}$ represented by Eq. (14) is expressed as

$$
\begin{aligned}
& \lim _{R^{\prime} \rightarrow \infty} \mathbf{u}_{0}^{\prime}=\frac{\alpha}{\alpha_{0}} \mathbf{e}_{y}\left[1-\alpha_{0}(3-4 \nu) \log \frac{\tilde{Q}_{2}+e M_{a}-\tilde{x}}{\tilde{Q_{1}}-e M_{a}-\tilde{x}}\right] \\
& \quad+\alpha \tilde{y}\left[\frac{1}{\tilde{Q_{1}}}-\frac{1}{\tilde{Q}_{2}}\right] \mathbf{e}_{x}+\frac{\alpha \tilde{y}}{\tilde{r}}\left(\frac{\tilde{x}-e M_{a}}{\tilde{Q}_{2}}-\frac{\tilde{x}+e M_{a}}{\tilde{Q}_{1}}\right) \mathbf{e}_{r}+O\left(\frac{1}{R^{\prime 3}}\right)
\end{aligned}
$$

where

$$
\mathbf{e}_{r}=\frac{\tilde{\mathbf{r}}}{\tilde{r}}=\frac{\tilde{y} \mathbf{e}_{y}+\tilde{z} \mathbf{e}_{z}}{\tilde{r}}, \quad \tilde{Q_{1}}=\left[\left(\tilde{x}-e M_{a}\right)^{2}+\tilde{r}\right]^{1 / 2}, \quad \tilde{Q_{2}}=\left[\left(\tilde{x}+e M_{a}\right)^{2}+\tilde{r}\right]^{1 / 2} .
$$

In outer variables the prolate spheroid reduces to a needle of zero radius and finite length when $M_{a}$ is arbitrary. We take the lead from hydrodynamics $[4,5]$ and assume that the end effects are negligible for the leading term of the outer expansion. Accordingly, as a first approximation, we still take $\mathrm{q}_{1}$ to be given by (19).

For $\tilde{r} \rightarrow 0$ and $-e M_{a}<\tilde{x}<e M_{a}$, the inner limit of $\mathrm{q}_{1}$ in the plane $\tilde{r}=0$ is given as

$$
\lim _{r \rightarrow 0} \mathbf{q}_{1}=\mathbf{e}_{y}\left[B^{*} L_{1}-\tilde{B} L_{2}+\left(B^{*}+\tilde{B}\right) \log \frac{\tilde{r}}{2}\right]-\frac{\left(B^{*}-\tilde{B}\right) \tilde{y} \mathbf{e}_{r}}{\tilde{r}}+O(\tilde{r} \log \tilde{r}),
$$


where

$$
\begin{aligned}
L_{1} & =\frac{1-\left(1+i e m_{a}\right) \exp \left(-i e m_{a}\right)}{\left(e m_{a}\right)^{2}}+\frac{1}{2}+\gamma-\operatorname{Ci}\left(e m_{a}\right)+i \operatorname{Si}\left(e m_{a}\right)+\log \frac{m_{a}}{M_{a}}, \\
L_{2} & =\frac{1-\left(1+i e M_{a}\right) \exp \left(-i e M_{a}\right)}{\left(e M_{a}\right)^{2}}+\frac{1}{2}-\gamma+\operatorname{Ci}\left(e M_{a}\right)-i \operatorname{Si}\left(e M_{a}\right), \\
C i(z) & =-\int_{z}^{\infty} \frac{\cos t}{t} d t ; \operatorname{Si}(z)=\int_{0}^{z} \frac{\sin t}{t} d t .
\end{aligned}
$$

The above value of $q_{1}$ must match with the value of the outer limit (26) at $\tilde{x}=0$; that is,

$$
\lim _{R^{\prime} \rightarrow \infty, t=0} u_{0}^{\prime}=\frac{\alpha}{\alpha_{0}} \mathbf{e}_{y}\left[1-2 \alpha_{0}(3-4 \nu) \log \frac{2 e M_{a}}{\tilde{r}}\right]-\frac{2 \alpha \tilde{y}}{\tilde{r}} \mathbf{e}_{r}+O\left(\frac{1}{R^{\prime 3}}, \tilde{r}^{2}\right) .
$$

When we match this value with that of $\mathbf{e}_{y}+\mathbf{q}$ from (27) we find that

$$
B^{*}=4 \alpha(1-\nu), \quad \tilde{B}=2 \alpha(1-2 \nu),
$$

and

$$
\alpha^{-1}=\alpha_{0}^{-1}-2\left[(3-4 \nu) \log e M a+2(1-\nu) L_{1}-(1-2 \nu) L_{2}\right] .
$$

The total force on the spheroid is now obtained by integrating the effect of the (constant-density) fundamental solutions of (12). Consequently,

$$
P=4 \pi \mu d_{0} \exp (i \omega t) \int_{-a e}^{a e} B^{*} d \xi=32 \pi \mu d_{0} a e \alpha(1-\nu) \exp (i \omega t)
$$

where $\alpha$ is given by (29). This is a new result and has some interesting consequences.

In the limiting case when $m_{a}$ tends to zero, (30) reduces to

$$
\begin{array}{r}
\lim _{m_{a} \rightarrow 0} P=32 \pi \mu d_{0} a e(1-\nu) \alpha_{0}\left[2+\frac{8}{3} e(1-\nu) \alpha_{0}\left\{2+\left(\frac{1-2 \nu}{2-2 \nu}\right)^{3 / 2}\right\} m_{a} i\right] \\
\cdot \exp (i \omega t)+O\left(m_{a}^{2}\right) .
\end{array}
$$

This limit agrees with formula (24) although we have not assumed this time that $m_{a}$ and $m_{b}$ are of the same order of magnitude. Accordingly, it can be applied also when $m_{b}$ is much smaller than $m_{a}$. Since the ratio $m_{b} / m_{a}$ is the same as the axis ratio $b / a, m_{b}$ being of the same order as $m_{a}$ means that the spheroid is nearly spherical. On the other hand, when $m_{b}$ is much smaller than $m_{a}$, the slenderness parameter $b / a$ is very small. Thus, when the spheroid becomes very slender $(b / a \rightarrow 0$ or $e \rightarrow 1)$, we derive the limit

$$
\lim _{m_{a} \rightarrow 0, e \rightarrow 1} P=\frac{16 \pi d_{0} a(1-\nu)}{\frac{1}{2}+(3-4 \nu) \log \frac{2 a}{b}}\left[1+\frac{4(1-\nu)\left\{2+\left(\frac{1-2 \nu}{2-2 \nu}\right)^{3 / 2}\right\} m_{a} i}{3\left\{\frac{1}{2}+(3-4 \nu) \log \frac{2 a}{b}\right\}}\right] \exp (i \omega t) .
$$

In the other limit, when $m_{a}$ is large and $e \rightarrow 1$, the spheroid becomes an infinitely long cylinder of radius $b$. Therefore, the force per unit length on an infinitely long cylinder of radius $b$ follows from $(30)$ as 


$$
\lim _{m_{a} \rightarrow \infty, e \rightarrow 1} \frac{P}{2 a}=\frac{8 \pi \mu d_{0}(1-\nu) \exp (i \omega t)}{(3-4 \nu)\left(\log \frac{2}{M_{b}}-\gamma-\frac{i \pi}{2}\right)-2 \log \frac{m_{b}}{M_{b}}} .
$$

In this paper our aim has been to present the first-order approximation and we have, accordingly, evaluated only the terms $u_{1}^{\prime}$ and $q_{1}$ of the expansions (6) and (10). For evaluating the higher-order terms the following word of caution is in order. (The authors are grateful to the referee for pointing it out to them.) Eq. (2a) is expressed in terms of a mixed description; that is, the spatial derviatives are based on the Eulerian description and the time derivatives are referred to the Lagrangian description. In order to pursue the present method for a higher-order approximation the term $\partial^{2} u / \partial t^{2}$ in $(3 a)$ should be replaced by its corresponding Eulerian form which relfects the change of reference frame.

\section{REFERENCES}

[1] R. P. Kanwal and D. L. Sharma, Singularity methods for elastostatics, J. Elasticity 6, 405-418 (1976)

[2] R. P. Kanwal, Dynamical displacements in an infinite elastic space and matched asymptotic expansions, J. Math. Physics 44, 275-283 (1965); 47, 222 (1968)

[3] R. P. Kanwal, Integral equations formulation of classical elasticity, Quart. Appl. Math. 27, 57-65 (1969)

[4] A. T. Chwang and T. Y. Wu, Hydrodynamics of low-Reynolds-number flow, part 4, J. Fluid Mech. 75, 677-689 (1976)

[5] Y. Y. Shi, Low Reynolds number flow past an ellipsoid of revolution of large aspect ratio, J. Fluid Mech. 23, 657-671 (1965) 